\title{
La estabilización articular en la técnica de la guitarra. El papel de Abel Carlevaro
}

\author{
The articular stabilization in guitar technique. \\ The role of Abel Carlevaro
}

\author{
por \\ Ricardo Barceló Abeijón \\ CEHUM. Universidade do Minho, Portugal \\ ricardobarcelo@ilch.uminho.pt
}

La estabilización de las articulaciones corporales, acto también conocido como fijación, es una constante en las múltiples acciones que realizamos en la vida cotidiana, habitualmente de forma instintiva e inconsciente. En la técnica guitarrística la fijación articular ha sido entendida normalmente como un recurso biomecánico que implica la estabilización firme, circunstancial y controlada, de una o más articulaciones corporales, en ocasiones de forma consciente y deliberada, en particular durante la fase de preparación de una obra musical. El guitarrista y pedagogo uruguayo Abel Carlevaro adoptó este concepto como uno de los pilares de su técnica instrumental. En este trabajo nos propusimos indagar, principalmente, acerca del origen del uso de las estabilizaciones articulares voluntarias en la técnica de la guitarra clásica y la hipotética influencia de los métodos con argumentación científica para la enseñanza del piano, publicados durante la primera mitad del siglo XX, tales como los de Otto Ortmann y Arnold Schultz. También investigamos los trabajos pedagógicos de los guitarristas Abel Carlevaro y Charles Duncan relacionados con esta temática, y el alcance que han tenido los mismos, con relación a la adopción de este concepto por la comunidad guitarrística internacional.

Palabras clave: Estabilización, fijación, guitarra, piano, Carlevaro, biomecánica, pedagogía.

The articular stabilization is an action known too as 'fixation' and it is quite common in the multiple actions that we carry out in everyday life, usually instinctively and unconsciously. In the guitar technique, fixation is normally understood as a biomechanical resource that implies the firm, circumstantial and controlled stabilization of one or more corporal articulations, sometimes consciously and deliberately, particularly during the preparation phase of a musical work. The Uruguayan guitarist Abel Carlevaro has adopted this concept as a fundament in his instrumental technique. In this paper we set out to investigate, mainly, the origin of voluntary fixations used in the classical guitar technique and the hypothetic influence of the methods with scientific argumentation for piano teaching, published during the first half of the 20th century, such as those of Otto Ortmann and Arnold Schultz in its development. We also investigate the pedagogical work of guitarists Abel Carlevaro and Charles Duncan related to this topic, and the scope they have had, in relation to the adoption of this concept by the international guitarist community.

Keywords: Stabilization, fixation, guitar, piano, Carlevaro, biomechanics, pedagogy. 


\section{INTRODUCGIÓN ${ }^{1}$}

Decía Bernardo de Chartres que somos como enanos a hombros de gigantes. Podemos ver más y más lejos que ellos, no por la agudeza de nuestra vista, ni por la altura de nuestro cuerpo, sino porque somos elevados por su gran altura ${ }^{2}$.

Juan de Salisbury. Metalogicon, 1159 (III, 4)

La estabilización de las articulaciones corporales es un acto habitualmente instintivo e inconsciente también conocido como fijación. El concepto de fijación en la técnica de la guitarra clásica es atribuido normalmente al guitarrista uruguayo Abel Carlevaro (Barceló 2000: 15; Fernández 2016). En la técnica guitarrística, la fijación ha sido entendida normalmente como un recurso biomecánico que implica la estabilización firme, circunstancial y controlada, de una o más articulaciones, en ocasiones de forma consciente y deliberada, en particular durante la fase de preparación de una obra musical. Se considera que uno de los aportes más novedosos de Carlevaro a la técnica de la guitarra fue, muy probablemente, la perspectiva teórico-práctica y la sistematización del uso consciente y voluntario de la estabilización funcional de las articulaciones, para conseguir diferentes resultados musicales y artísticos, así como la optimización ergonómica de los movimientos, entre otros.

En este trabajo nos propusimos indagar, entre otras cuestiones, si este es realmente un concepto original de Carlevaro, es decir, si la elaboración de la teoría de las fijaciones fue un proceso intelectual individual que él mismo desarrolló, o si se basó en ideas de otros autores. Consideramos interesante investigar en este sentido, ya que existen antecedentes de una inexacta atribución a Abel Carlevaro del mérito de ser el primer guitarrista que tuvo la preocupación de evitar ciertos ruidos producidos por los dedos de la mano izquierda al abandonar las cuerdas de la guitarra, tanto como de la forma de conseguirlo, uno de los

1 Este texto no forma parte de otro proyecto de mayor alcance. De hecho, este fue redactado como un artículo independiente, basándome en conocimientos adquiridos directamente en clases particulares de guitarra con Abel Carlevaro (1916-2001) -creador de una "escuela" guitarrística innovadora en el siglo XX-, y con sus exalumnos Eduardo Fernández, en Montevideo, y Miguel Angel Girollet, en Madrid, entre 1984 y 1990. A pesar de que estos conocimientos fueron muy valiosos para mi evolución como intérprete, surgieron ciertas inquietudes respecto de algunos puntos específicos, en particular en torno al concepto de fijación, uno de los puntos fundamentales de las enseñanzas de Carlevaro, que me llevaron a investigar en este terreno particular de la técnica de la guitarra clásica. Considero que la investigación realizada llena algunas lagunas en la historia de la interpretación y la técnica de la guitarra clásica, y esta ha sido la principal motivación para escribir este estudio. También espero que el mismo pueda ser un aliciente para que algunos miembros de la comunidad guitarrística puedan conocer mejor el recurso de la estabilización articular en la técnica de la guitarra.

2 Texto original: Dicebat Bernardus Carnotensis nos esse quasi nanos, gigantium humeris incidentes, ut possimus plura eis et remotiora videre, non utique proprii visus acumine, aut eminentia corporis, sed quia in altum subvenimur et extollimur magnitudine gigantea. Todas las traducciones que aparecen en este artículo son de nuestra autoría. 
preceptos más populares de su escuela ${ }^{3}$. En realidad, el guitarrista español Dionisio Aguado ya había tratado de este asunto más de un siglo antes ${ }^{4}$ :

Teniendo los dedos de esta mano siempre abiertos (fig. 7, lám. $2^{\mathrm{a}}$ ) resulta la ventaja de que al levantarse ellos de la cuerda pisada lo hace cada uno paralelamente a la división, y se evita un ruido o silbido sutil que el dedo produce al retirarse especialmente de los bordones, el cual se verifica cuando los dedos se levantan en dirección diagonal a la cuerda (Aguado 1843: 25).

Entonces nos preguntamos ¿no podría haber habido un error similar en la atribución de la creación de la teoría de las fijaciones?

Para corroborar cuál fue la vía de introducción de las fijaciones conscientes con objetivos definidos en la técnica de la guitarra, pretendimos saber cómo surgió este enfoque técnico plasmado en Escuela de la Guitarra (1979) de Abel Carlevaro y en la Serie Didáctica para Guitarra (1966-1975), del mismo autor -la que cuenta con cuatro cuadernos de técnica-, y cómo fue el proceso de adopción de tal concepto. También quisimos averiguar si este músico se apoyó en algún tipo de material bibliográfico o científico durante el proceso de elaboración de la teoría de las fijaciones, en qué momento comenzó el mismo, y cuándo quedó pronto el sistema para su aplicación práctica y pedagógica.

Consideramos también importante para nuestro estudio conocer el nombre de los primeros guitarristas que adoptaron cabalmente este concepto en su técnica, cómo comenzó la difusión del uso voluntario de la fijación y qué alcance y aceptación ha tenido en el ambiente guitarrístico, desde el principio de su utilización intencional hasta hoy, para saber hasta qué punto la teoría de las fijaciones es conocida y aceptada por la comunidad guitarrística.

Durante la redacción del presente artículo pensamos que era importante para nuestra investigación también conocer, de primera fuente, la opinión de algunas de las personas que tuvieron un contacto temprano con las preocupaciones pedagógicas de Carlevaro en torno a la enseñanza de la guitarra clásica (Escande 1996 y 2019; Primus y Zeoli 1997; Jiménez Muñoz 2013: 27). Para eso, elaboramos un cuestionario que abordó los principales asuntos que exponemos en esta introducción, el que fue respondido por músicos que nos brindaron información muy valiosa para comprender mejor el proceso de inclusión de las fijaciones como uno de los recursos de la técnica carlevariana. Los datos obtenidos en tal cuestionario fueron analizados para llegar a nuestras conclusiones, y así, algunos fragmentos extraídos de las respuestas recibidas son citados a lo largo de este trabajo.

3 Es muy interesante considerar el comentario de Magdalena Gimeno, quien fue alumna de Carlevaro entre 1967 y 1982: "Carlevaro hablaba mucho más de toques y búsqueda de sonido que propiamente de la fijación, aunque para todo lo que se hace con un instrumento la fijación está presente. La insistencia en usar la palabra "fijación”, así como otros puntos de su teoría, se fue haciendo más notoria cuando empezó a salir del país (Uruguay) a dar seminarios. Tal vez por la sorpresa que eso causó, no lo puedo saber, solamente hacer alguna suposición. Es como la idea de evitar el ruido con las cuerdas en la mano izquierda. Fue tomando una importancia en la que no insistía de esa manera. Lo que le importaba era el resultado musical de algo y la claridad" (Magdalena Gimeno, comunicaciones personales por e-mail, 13 y 14 de septiembre, 2018).

4 No obstante, consideramos que la asimilación de esta idea al corpus de elementos técnicos incorporados a la "Escuela de Carlevaro" no le quita en absoluto valor a la misma, por el contrario. Carlevaro agrega que es necesario algún grado de fijación de los dedos de la mano izquierda para que el brazo pueda separarlos convenientemente de las cuerdas, evitando así la fricción indeseada y el ruido resultante, explicitando los procedimientos necesarios para que la acción sea eficaz, lo que fue totalmente nuevo en la técnica guitarrística. 


\section{DEFINICIÓN DE FIJACIÓN}

Carlevaro define la fijación como la anulación voluntaria y momentánea de una o varias articulaciones, con la finalidad de permitir que los elementos más adecuados puedan actuar para conseguir un determinado objetivo, y agrega que:

La fijación es entonces un acto voluntario que controla una determinada articulación y la lleva a un cierto estado de anulación momentánea para permitir la transmisión de un movimiento o fuerza a través de ella, que sirve así de puente o nexo [...] Con el uso consciente y selectivo de las FIJACIONES puede producirse un cambio radical anulando la fatiga inútil [...] (Carlevaro 1979: 34).

Este autor, en la misma publicación, afirma que "cuando un dedo no puede realizar con naturalidad y holgura cierto movimiento o movimientos, debe delegarse (por medio de fijaciones voluntarias) a otra parte del complejo mano-brazo la ejecución del correspondiente mecanismo o pasaje" (Carlevaro 1979: 35).

Sin embargo, teniendo en cuenta las consideraciones de especialistas del campo de la biomecánica y de la interpretación musical, pensamos que Carlevaro tal vez no utilizó el término más adecuado para definir la acción que él denominó fijación, lo que también sucedió con la adopción del vocablo distensión, que el guitarrista uruguayo escogió para identificar lo que es denominado como extensión por muchos otros autores, ya que el término distensión hace referencia, por lo menos en medicina, a una patología muscular. Carlevaro no explicó cómo se produce la fijación desde el punto de vista anatomofisiológico, pero tal vez habría sido más adecuado que hubiera utilizado el término estabilización, y no fijación, como se deduce de la explicación dada en el libro de medicina deportiva El cuerpo y sus movimientos. Bases científicas (Gowitzke y Milner 1999), del que citamos algunos pasajes a continuación para sostener nuestro punto de vista.

Cuando una articulación se fija voluntariamente, en vez de estabilizarse, hay, además de la inmovilización, una rigidez resultante de la fuerte contracción isométrica de todos los músculos que cruzan dicha articulación. Estos músculos resistirán intensamente todos los esfuerzos exteriores por desplazar esa articulación [...] uno habría de ser capaz de reconocer la diferencia entre la estabilización y la fijación de las articulaciones. Como ya hemos dicho, la fijación denota una rigidez en oposición a todo movimiento, mientras que la estabilización sólo implica firmeza. La fijación se puede producir sin que el sujeto se aperciba de ella $[\ldots]$ La economía de movimiento implica el uso de una sinergia de estabilización y la evitación de la fijación de las articulaciones. Los consejos en la enseñanza deben ir dirigidos a este fin [...] (Gowitzke y Milner 1999: 171).

La utilización de la palabra fijación para describir la sinergia de estabilización de las articulaciones, provocada por las fuerzas concurrentes que representan los músculos agonistas y antagonistas (Fernández 2000: 23), y cuyo objetivo es, efectivamente, neutralizar la movilidad de una articulación de forma funcional, no ha sido probablemente la mejor opción, ya que puede inducir a una mala interpretación, como se constata en esta declaración de Carlevaro:

[...] la fijación es de vital ayuda para una movilidad dócil y positiva, y no debe tomarse erróneamente como rigidez (Escande 2019: 228).

No obstante, y a pesar de que el término fijación ha trascendido hasta cierto punto en el ámbito de la técnica de la guitarra y del piano, no consideramos conveniente mantener 
su uso actual para definir el procedimiento descrito, porque su etimología no es la más precisa $^{5}$. Por tanto, sugerimos la adopción del vocablo estabilización en lugar de fijación, y de las palabras equivalentes en otros idiomas, para conseguir una mayor precisión en el lenguaje, lo que podrá facilitar la comunicación entre músicos y especialistas de áreas como la biomecánica, la medicina y la motricidad humana.

Un trabajo académico realizado por el investigador canadiense Wheatley-Brown (2001) revela, de forma muy acertada, ciertas dificultades de comunicación en la pedagogía músicoinstrumental, lo que resulta muy pertinente para nuestro estudio, como podremos ver en la siguiente citación. Se destaca un uso problemático de la terminología en la enseñanza del piano en general, que también incluye al término fijación.

La extensa recopilación de datos del lenguaje utilizado por los autores seleccionados reveló problemas significativos derivados del uso de un lenguaje inconsistente e inexacto para describir y definir conceptos básicos. Un análisis del lenguaje encontró varias razones a las que atribuir tal inconsistencia e inexactitud: hay una comprensión incompleta de los principios anatómicos y biomecánicos; los autores luchan para conciliar las dualidades opuestas de estabilidad y flexibilidad que tipifican gran parte de la técnica del piano ${ }^{6}$ (Wheatley-Brown 2001: 118).

En la enseñanza de instrumentos musicales es frecuente la utilización de un lenguaje metafórico como forma de comunicación, junto con una mezcla de términos de origen heterogéneo, eventualmente útiles para transmitir ideas y emociones en el terreno de la expresión musical, lo que puede ser equívoco cuando se dan indicaciones del ámbito de la biomecánica ${ }^{7}$.

[...] Los autores no siempre distinguen lo que sienten del hecho real y mezclan libremente terminología científica, común e inventada. En lugar de avanzar e iluminar nuestra comprensión de la técnica del piano, este lenguaje problemático a menudo desinforma y confunde ${ }^{8}$ (WheatleyBrown 2001: 118).

5 Como veremos a lo largo de este trabajo, han existido varias expresiones para identificar al mismo recurso técnico, lo que da la pauta de su importancia. Estas son algunas de ellas: anclaje, bloqueo, besactivación, estabilización, fijación, firmeza, inmovilización, rigidez.

6 Texto original: The extensive data collection of the language used to describe and define core concepts from the selected authors revealed significant problems with inconsistent and inaccurate language. An analysis of the language found various reasons that can be attributed to inconsistency and inaccuracy: there is an incomplete understanding of anatomical and biomechanical principles; the authors struggle to articulate the opposing dualities of stability and flexibility that typifies much of piano technique.

7 Como forma de destacar la importancia de conocer los principios que refiere Wheatley-Brown, tanto en el piano como en la guitarra, utilizaremos una analogía: ciertos artistas plásticos han adquirido conocimientos de anatomía con la finalidad de representar figuras humanas adecuadamente, porque, aunque los músculos, tendones y huesos no se puedan ver directamente desde el exterior del cuerpo, sí se pueden ver sus formas y movimientos, que se manifiestan en la topografía de la piel. Sin embargo, y a pesar de que esto no sea estrictamente necesario, el conocimiento de esas estructuras ocultas tendrá, seguramente, influencia positiva en la imaginación del artista en el momento de esculpir o pintar una figura humana de forma realista y convincente. De la misma forma, un músico que se apoye en sólidos conocimientos anatomofisiológicos y biomecánicos podrá tener, seguramente, más elementos para elaborar metáforas eficaces y escoger los términos más pertinentes para utilizar como herramientas en sus clases.

8 Texto original: [...] the authors do not always distinguish feeling from fact; and the authors freely interchange scientific, common, and invented terminology. Rather than advancing and enlightening our understanding of piano technique, this problematic language often misinforms and misleads. 
Consideramos que, aunque ciertas figuras retóricas puedan servir para transmitir adecuadamente algunas ideas artísticas musicales apoyándose en una vivencia subjetiva, los recursos técnico-mecánicos que son necesarios para concretizarlas deberían ser identificados perfectamente, como forma de emitir los mensajes adecuadamente y evitar malentendidos.

[...] a menudo, esta amplia terminología contribuye a la confusión. Se opta frecuentemente por el uso de diferentes términos, y así, los conceptos adquieren diferentes significados dependiendo de cómo estos son utilizados. Existe poco consenso en torno a la manera en que los conceptos contribuyen a la técnica del piano, dando como resultado consejos contradictorios ${ }^{9}$ (WheatleyBrown 2001: 120-121).

Es posible entonces llegar a la conclusión de que tanto los términos específicos como el lenguaje figurativo deben corresponder correctamente con las ideas que se quieren transmitir, para conseguir los objetivos pretendidos, como sostiene Wheatley-Brown.

Si se promueve la idea de que la técnica del piano debe estar basada en principios anatómicos y biomecánicos, entonces la comunidad pedagógica tiene la obligación de garantizar que el lenguaje utilizado refleje esto con precisión ${ }^{10}$ (Wheatley-Brown 2001: 122).

Son varios los pianistas, como se puede extraer del estudio realizado por WheatleyBrown, que han tenido la percepción de que el término fijación no es adecuado. También hemos observado que el vocablo estabilización (stabilization), dentro de una variedad de denominaciones, ha sido usado por varios autores prestigiosos de los últimos cincuenta años para identificar esa acción específica. Han sido esas, principalmente, las razones fundamentales de que en el título del presente trabajo nos hayamos decantado por la denominación estabilización articular y no fijación. Consideramos, además, que estabilización es una denominación correcta desde el punto de vista científico, pues lo que define es un grado variable de co-contracción de músculos agonistas y antagonistas para conseguir una tensión muscular relativamente estática, gracias a un equilibrio de fuerzas, siendo de utilidad identificar oportunamente las articulaciones que se deben estabilizar para conseguir los resultados musicales pretendidos.

Por otra parte, vemos que, en sus escritos didácticos, Carlevaro no se refiere a la variabilidad del grado de una fijación/estabilización. Sin embargo, el guitarrista y pedagogo estadounidense Charles Duncan, quien escribió el libro Art of classical guitar playing (1980), y que tal como Carlevaro utilizó la denominación de fijación (fixation) para denominar al recurso del que tratamos, sí menciona la posibilidad de conseguir grados de tensión variable con el uso de las fijaciones, lo que es importante para lograr un mejor entendimiento de la acción:

9 Texto original: [...] it is this wide range of terminology that often contributes to the confusion. Frequently switching between the terms, the concepts take on different meanings depending on how it is being used. There is little consensus in how the concepts contribute to piano technique, resulting in conflicting advice.

10 Texto original: If piano technique is promoted as being based on anatomical and biomechanical principles, then the pedagogical community has an obligation to ensure that the language they use accurately reflects this. 
El grado exacto de tensión empleado en la articulación de la punta del dedo es una buena cuestión. [...] Varía entre diferentes ejecutantes, entre diferentes dedos de la misma mano. O hasta en una misma mano y dedo, según el contexto musical. [...] El grado de flexibilización está determinado por la sensación cinestésica ${ }^{11}$ (Duncan 1980: 6-7).

Sabemos que Duncan, guitarrista y pedagogo, era conocedor del trabajo previo de Carlevaro, porque los cuadernos 3 y 4 (1969-1975) aparecen como referencias bibliográficas en el libro del músico norteamericano, publicado un año más tarde que Escuela de la Guitarra del uruguayo. Podemos deducir que Duncan adoptó sin muchos cuestionamientos el término fijación por ya haber sido utilizado en el vocabulario guitarrístico por Abel Carlevaro, pero principalmente por influencia de los escritos del pianista Arnold Schultz, al que Duncan hace referencia en su publicación, de cuyo influyente trabajo hablaremos más adelante.

\section{EL RECURSO DE LA ESTABILIZACIÓN ARTICULAR EN LA TÉCNICA GUITARRÍSTICA}

Todos los guitarristas utilizan la estabilización articular como un recurso técnico durante la ejecución instrumental, algunos de manera consciente y deliberada, y otros de forma instintiva. Porque "el único estado en que una persona viva no tiene articulaciones fijadas ni músculos en tensión es cuando ella está inconsciente"12 (Duncan 1980: 1).

En realidad, las estabilizaciones articulares son omnipresentes en nuestra vida diaria, así como lo expone claramente Alfredo Escande. Las fijaciones son "[...] procedimientos que forman parte de nuestro bagaje de movimientos útiles en todas las situaciones de la vida cotidiana (desde levantar un lápiz, un libro o una garrafa de supergás, hasta batir un huevo, pegarle a una pelota de fútbol o señalar el horizonte)" (Alfredo Escande, comunicaciones personales por e-mail, 10 de junio y 24 de agosto, 2018).

Las estabilizaciones controladas tampoco son raras en las artes marciales, en las que se utilizan para obtener una mayor fuerza de impacto. El puño golpea, pero en realidad, una cadena de estabilizaciones articulares permite que la fuerza muscular de las piernas y del tronco llegue al extremo del brazo.

Un ejemplo proveniente de la técnica instrumental es cuando se toca utilizando el apoyando tradicional, donde se estabilizan normalmente las articulaciones de la falangina y la falangeta, para que el movimiento pueda ser efectuado utilizando como base la articulación del nudillo, aunque el guitarrista no tenga consciencia de lo que acontece desde el punto de vista fisiológico. Ese tipo de estabilizaciones en las articulaciones de los dedos también se produce cuando se utiliza un mecanismo conocido por un buen número de guitarristas de la península ibérica como falso apoyo, pero no utilizando la cuerda contigua como freno para el ataque, sino los músculos extensores que detienen el movimiento de flexión del dedo, consiguiendo una calidad de sonido idéntica al del apoyando si el ataque es realizado adecuadamente. Este es uno de los aspectos de las estabilizaciones como medio para controlar la calidad y el volumen sonoro en la guitarra. Ahí radica su importancia y la necesidad de identificar su uso, tanto para mejorar el control en la ejecución, como para facilitar la transmisión de su forma de aplicación.

11 Texto original: The exact degree of tension employed at the tip joint is a nice question. [...] It varies between different players, between different fingers on the same hand. Or even for the same hand, or even for the same finger depending on musical context. [...] The amount of "give" is determined by kinesthetic sensation.

12 Texto original: The only living state in which your joints are not fixed and muscles tensed is when a person is unconscious. 
También es de destacar que es común en el pianismo moderno estabilizar las articulaciones del codo, de la muñeca, y hasta las de los dedos, utilizando el hombro como eje del movimiento, para obtener una palanca de mayor longitud, una masa mayor y, consecuentemente, mayor fuerza al presionar la tecla con el brazo entero funcionando como unidad, lo que es necesario para obtener un ataque de gran potencia sonora. En palabras de Schultz: "Cuando los músculos antagonistas ponen firmes las articulaciones que están después del punto de apoyo, estas se sueldan como si formaran una sola pieza con las otras partes"13 (Schultz 1949: 63). Según este mismo autor, las articulaciones que se utilizan para el ataque en la mecánica instrumental funcionan como una palanca de tercer género (Schultz 1949: 65), lo que se hace evidente ante la resistencia que representa una cuerda o una tecla, cuestión física que no fue identificada por Carlevaro, pero que puede ayudar a entender mejor los diferentes tipos de ataque.

Es sabido que una palanca sirve para amplificar la fuerza mecánica aplicada a un objeto, desde que se cuente con un punto de apoyo. Si consideramos que cuanto mayor longitud tenga una palanca, más eficiente será el esfuerzo realizado para mover un objeto, entonces las estabilizaciones articulares, además de pasar la responsabilidad del esfuerzo necesario a músculos más competentes, cumplirían también la función de ir aumentando la longitud de las palancas biomecánicas representadas por la "anulación" del funcionamiento de diferentes articulaciones, básicamente de los dedos o de los brazos. Así, se van uniendo por estabilización los diferentes segmentos de los miembros o de los dedos -según las necesidades de la ejecución instrumental-, que se sirven de una articulación en particular como punto de apoyo para ejercer fuerza sobre un objeto -la resistencia-, que puede estar representado, por ejemplo, por una cuerda.

Duncan va un paso más allá que Carlevaro y adapta las ideas de Schultz acerca de la estabilización de las articulaciones y la acción de los dedos en forma de palanca aplicándolas a la técnica guitarrística, como se puede ver en el siguiente gráfico (ver Figura 1).

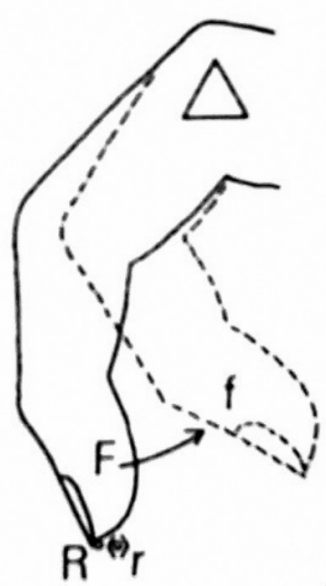

Figura 1. Duncan 1980: 6.

13 Texto original: When antagonistic muscles stiffen the various joint of the base, it is welded as if into a single piece with the body. 


\section{ORIGEN DEL CONCEPTO DE ESTABILIZACIÓN ARTICULAR EN LA GUITARRA}

La introducción de este concepto en la técnica de la guitarra parece deberse a Abel Carlevaro, aunque pudiera tratarse de una adaptación a este instrumento y no una creación original, lo que quisimos indagar. Los guitarristas entrevistados ${ }^{14}$ respondieron de la siguiente manera con relación al origen del concepto de fijación:

Escande: No sé de otro teórico de la guitarra que haya planteado, antes de Carlevaro, este concepto. [...] la idea -en cuanto a su aplicación a la técnica guitarrística- es original de Abel Carlevaro. No sé si es necesario aclarar que, en cuanto a las acciones (llamémosle, desde mi condición de neófito en ciencias biológicas) neuromusculares, él no inventó nada, sino que observó -con la agudeza y el sentido lógico que lo caracterizaba- [...] (Alfredo Escande, comunicaciones personales por e-mail, 10 de junio y 24 de agosto, 2018).

Fernández: En la literatura guitarrística no conozco otros ejemplos, a menos que se considere el apoyado como una fijación implícita, lo que de hecho ocurre. En la técnica pianística, según mi información, aparece prácticamente desde Beethoven, aunque no siempre explicitada (Eduardo Fernández, comunicación personal por e-mail, 8 de agosto, 2018).

Gimeno: El trabajo de Carlevaro fue observar esto y explicarlo, no "inventarlo". Él contaba que una vez asistió a una clase de Rachmaninoff ${ }^{15}$ y al explicar cómo destacar unas notas, aunque sin usar la palabra "fijación", lo que hacía era eso (Magdalena Gimeno, comunicaciones personales por e-mail, 13 y 14 de septiembre, 2018).

Atendiendo a los comentarios de los tres entrevistados, podríamos deducir que el concepto de fijación era preexistente en la técnica pianística, aunque fuera de forma implícita, y que Carlevaro lo asimiló en su técnica, siendo el primer guitarrista en adaptarlo a la guitarra clásica.

Continuando con nuestras entrevistas, preguntamos también en qué año consideraban que la teoría quedó a punto para su aplicación práctica y pedagógica, a lo que respondieron:

Escande: Creo que Carlevaro fue desarrollando el concepto de fijación a medida que iba meditando y elaborando su teoría (podríamos decir que entre principios de los cincuenta, cuando empezó a escribir sus ideas en borrador y principios de los setenta, cuando empezó a exponerlas). Cuando yo lo conocí, en 1974, la mayoría de sus conceptos estaban ya consolidados (aunque algunos terminaron de afinarse en el proceso de escritura del libro) (Alfredo Escande, comunicaciones personales por e-mail, 10 de junio y 24 de agosto, 2018).

Fernández: Seguramente al menos en el momento de la publicación de los Cuadernos (especialmente el $\mathrm{N}^{\circ} 2$ ), o más bien mucho antes, dado que los Cuadernos son simplemente la

14 Los guitarristas y pedagogos uruguayos Magdalena Gimeno (n. 1950), Alfredo Escande (n. 1949) y Eduardo Fernández (n. 1952), quienes comenzaron a estudiar con Abel Carlevaro en 1967, 1974 y 1972, respectivamente, fueron próximos del Maestro y conocieron, de primera mano, todos los detalles de su escuela desde una fase bastante temprana de elaboración y difusión. Agradecemos su valiosísima colaboración y su disponibilidad para responder nuestro cuestionario, que contenía varias preguntas útiles para nuestro estudio, aportando informaciones muy importantes para la elaboración de este artículo. También agradecemos los comentarios de Gonzalo Solari (n. 1955), y la lectura atenta de este trabajo de Manuel Gómez Ortigosa (n. 1958). Ambos tuvieron contacto con Carlevaro en una etapa posterior.

15 Según comentarios, al respecto, de Eduardo Fernández (que recibimos por e-mail el 29 de noviembre de 2018), podemos inferir que Carlevaro podría estar refiriéndose realmente al pianista Walter Gieseking (1895-1956). 
racionalización de una técnica (o más precisamente, un mecanismo) que Carlevaro venía aplicando desde mucho antes en su práctica personal como intérprete (Eduardo Fernández, comunicación personal por e-mail, 8 de agosto, 2018).

Gimeno: Conocí a Carlevaro en 1967. En ese momento tenía editado el libro de las escalas y completamente planeados los siguientes y el libro de teoría (Magdalena Gimeno, comunicaciones personales por e-mail, 13 y 14 de septiembre, 2018).

Teniendo en consideración las citaciones anteriores, existen notorios indicios de que la aplicación guitarrística de las estabilizaciones articulares -cuya madurez se podría situar alrededor de 1965- sea original de Carlevaro, pero no hay evidencia de que el concepto sea original de este autor.

Después de conocer algunas de las publicaciones del pianista inglés Tobias Matthay (1858-1945), y de los pianistas estadounidenses Otto Ortmann (1889-1979) y Arnold Schultz (1903-1972), vemos que en ellas se utilizó ampliamente el término fijación (en inglés: fixation), con la misma intención que Carlevaro. Teniendo en cuenta que las fechas de publicación de dichas obras (1929-1949) son anteriores y cercanas a los principios de la adopción de Carlevaro de su nueva técnica, comenzamos a preguntarnos si Carlevaro podría haberse basado en las ideas de estos pianistas, o si, por el contrario, la elaboración de su teoría de las fijaciones fue, de hecho, un proceso intelectual individual que él fue desarrollando de manera independiente.

En el supuesto de que Carlevaro hubiera conocido los escritos e ideas de Matthay, creemos que es poco probable que se inspirara en ellos, porque el pianista inglés solamente habla de la fijación para denostarla radicalmente (por lo menos como denominación) y, por tanto, su obra no parecería un buen modelo que seguir.

En lo que respecta a una hipotética influencia de Ortmann o de Schultz, a pesar de que el último acepta que pudiera haber un uso problemático de la fijación en la técnica del piano, defiende ampliamente las virtudes de una utilización adecuada de la misma en la ejecución instrumental artística. Considerando esto, pensamos que sería verosímil que las ideas presentadas en el libro de Ortmann The phisiologycal mechanics of piano technique, de 1929, o en The riddle of the pianist's finger de Schultz, publicado en 1936, pudieran haber sido una fuente de inspiración para Carlevaro. Sin embargo, si el guitarrista uruguayo se hubiera inspirado directa y efectivamente en los libros mencionados, habría utilizado seguramente una buena cantidad de argumentos científicos acerca del uso de la estabilización articular en la guitarra, ofreciendo datos biomecánicos, describiendo los grupos musculares involucrados y utilizando probablemente un modelo que explique el funcionamiento de las palancas en el cuerpo humano, como lo hicieron Ortmann, Schultz, y más tarde Duncan, para dar una base más sólida a sus estudios. Carlevaro no hizo esto, lo que debilita esta hipótesis.

Existe otra cuestión importante que puede dar pistas para dilucidar potenciales influencias. Carlevaro se refiere en sus libros a las ventajas de tocar en estado de relax-un factor que está relacionado con las fijaciones y el que abordaremos al final de este estudio-, pero como una forma de proceder general e indiferenciada, y no como el efecto de una coordinación muscular eficaz, interpretación que ya había sido criticada por los pianistas norteamericanos citados. De hecho, Schultz es categórico en este punto: "Adoptar el concepto de fijación es, sin embargo, romper abiertamente con el dogma de la relajación"16 (Schultz 1949: 62).

Si a todo esto sumamos que, en respuesta a nuestras preguntas, Escande y Fernández consideran que Carlevaro no se basó en otros autores para elaborar la teoría de las fijaciones

16 Texto original: To embrace the concept of fixation, however, is to break openly with the relaxation dogma. 
aplicada a la técnica de la guitarra clásica, podríamos especular entonces que Carlevaro llegó de forma individual a sus conclusiones. En ese caso, habría habido una convergencia de ideas -como ha sucedido ocasionalmente en el campo de la investigación y la ciencia-17, aplicadas a la técnica de dos instrumentos distintos (el piano y la guitarra), ideas que enriquecieron de forma evidente la formación de los guitarristas y su modo de abordar el estudio de las obras musicales a partir de la segunda mitad del siglo XX.

Ante la duda, le damos voz al propio Abel Carlevaro en esta discusión. En el siguiente extracto, Carlevaro habla de un guitarrista popular del Montevideo de su juventud, que atacaba las cuerdas con mucha agilidad utilizando solamente el pulgar, y cuenta que se inspiró en esa forma de utilizar la mano derecha para elaborar su concepto de fijación:

Entre otros, estaba el Petiso Tripaldi, que tocaba sólo con el pulgar, pero tan rápido, que yo no lo podía imitar. Yo a menudo lo observaba tocar, hasta que descubrí que él fijaba enteramente su pulgar. Fue de allí que pude encontrar mi concepto de "fijación", un descubrimiento muy importante para mí (Escande 2019: 138).

No debemos descartar, sin embargo, la posibilidad de que Carlevaro hubiera recibido alguna información indirecta respecto de este tema, ni tampoco una asimilación consciente o inconsciente, teniendo en cuenta el rico ambiente cultural que lo rodeaba en el momento en que desarrollaba sus ideas, y que entre sus relaciones contaba con numerosos conocidos del mundo de la música. Podemos imaginar que entre ellos habría, con toda seguridad, conocedores de los métodos más recientes de enseñanza del piano, que pudieran haber transmitido los avances técnicos de Ortmann y Schultz, dos grandes de la pedagogía del piano en el siglo $\mathrm{XX}^{18}$.

Podemos concluir entonces que Carlevaro no fue el primer instrumentista en utilizar el recurso técnico de la estabilización articularconsciente y voluntaria, ni en llamarlo fijación, pero, con toda seguridad, fue el primer guitarrista que lo usó. Fue esencial y muy importante el trabajo que realizó Carlevaro, buscando la forma de mejorar la técnica guitarrística gracias a una nueva manera de entender los elementos biomecánicos implicados en la ejecución, y la correlación fisiológica y musical entre los diferentes procedimientos seleccionados.

Los primeros estudiantes de guitarra que siguieron los preceptos de Carlevaro orientados por la fijación consciente y voluntaria, como uno de sus conceptos más importantes, fueron, según los datos que poseemos actualmente, algunos de sus primeros alumnos en el Conservatorio Nacional de Montevideo, Uruguay, así como comenta Escande:

17 Como sucedió, por ejemplo, con la Teoría de la Evolución: Darwin y Wallace llegaron a ideas similares de manera independiente por diferentes caminos, pero en la misma altura histórica, convergiendo hasta cierto punto en los resultados de sus estudios.

18 Solamente a título de ejemplo, es interesante recordar que en 1943 el Instituto Interamericano de Musicología, dirigido entonces por Francisco Curt Lange, organizó una Embajada Artística Uruguaya que visitó diferentes ciudades de Brasil en misión oficial. Estaba encabezada por Guido Santórsola (1904-1994) e integrada también por Carlevaro y las destacadas pianistas Sara Bourdillón de Santórsola (1901-1985) y Fanny Ingold (1922-1995). Santórsola era ciudadano italiano, nacionalizado uruguayo en 1936, con quien Carlevaro colaboró en diferentes oportunidades, orientando al compositor en las posibilidades de la guitarra. La pianista Sara Bourdillón era la esposa de Santórsola, y Fanny Ingold su alumna. En ese mismo viaje, durante su estadía en Río de Janeiro, Carlevaro tuvo la oportunidad de relacionarse con Heitor Villa-Lobos, quien también era pianista (Escande 2015: 143-144). Todo esto sin mencionar las experiencias que pueda haber tenido Carlevaro durante los viajes que realizó a Europa y a Estados Unidos, a partir de 1948. 
Creo que, de sus primeros alumnos en el Conservatorio Nacional (donde empezó a enseñar en 1959), aquellos que estudiaron con él en la segunda mitad de los años sesenta recibieron y adoptaron cabalmente ese concepto ya elaborado (Alfredo Escande, comunicaciones personales por e-mail, 10 de junio y 24 de agosto, 2018).

Fue fundamental la labor llevada a cabo entonces por Carlevaro para transmitir su sistema de enseñanza, dándole especial preponderancia al control de la estabilización de ciertas articulaciones para conseguir una técnica eficaz y fisiológicamente eficiente, lo que se reveló extremamente funcional gracias a los excelentes resultados obtenidos por sus alumnos. Algunos de los primeros guitarristas que tuvieron conocimiento de la aplicación consciente y voluntaria de las estabilizaciones articulares en la técnica de la guitarra fueron Baltazar Benítez (1944-2018), César Amaro (1948-2012) y Magdalena Gimeno (n. 1950). Un poco más tarde, Carlevaro también tuvo alumnos privados de la talla de Eduardo Fernández y de Álvaro Pierri.

\section{RELAJACIÓN Y ESTABILIZACIÓN}

Existe una relación dinámica entre la estabilización de las articulaciones y la relajación de los músculos durante la ejecución. Por esa razón dedicaremos una parte de este artículo a examinar diferentes opiniones acerca del relax, o la relajación durante la ejecución del instrumento. Hemos extraído algunos fragmentos del libro de Ortmann (1929) que citamos a continuación, para ilustrar más claramente la problemática, en especial por la importancia que no solo revela en el piano ${ }^{19}$, sino también en la ejecución guitarrística.

Sabemos que, normalmente, con cada impulso para que un músculo flexor se contraiga, se produce un impulso hacia el extensor correspondiente para relajarse. Es decir, tal es el caso si se desea mover (en este caso, flexionar) una articulación. Pero si se desea mover una articulación más distal, o se debe superar otra resistencia importante, los flexores y los extensores se contraen simultáneamente, estabilizando así la articulación firmemente contra el movimiento, y permitiéndole actuar como un punto de apoyo fijo necesario. El grado de rigidez depende del grado de contracción simultánea ${ }^{20}$ (Ortmann 1929: 57).

Como veremos enseguida, Ortmann se muestra poco satisfecho con el uso inadecuado de la palabra "relajación" en la técnica instrumental.

19 Como ya afirmamos en O Sistema Posicional na Guitarra (Barceló 2015), no tenemos reparos en recurrir a la técnica del piano para referirnos a problemáticas similares en la guitarra, "porque hemos observado que, en relación con los aspectos técnico-mecánicos, existe un funcionamiento intrínseco de las manos que revela una subyacente transversalidad técnico-instrumental. Con esto queremos decir que existen mecanismos potencialmente trasplantables de la técnica de uno a otro instrumento musical, si son debidamente adaptados. La organología de los instrumentos no es solo el fruto de necesidades acústicas y musicales, sino también es absolutamente deudora de las características anatómicas y fisiológicas de las manos y de otras partes del cuerpo humano implicadas en la ejecución. Por este motivo, los instrumentos siempre tienen algún punto en común en su técnica y en su construcción, por mínimo que sea, salvando las diferencias aparentes y superficiales.

20 Texto original: We know that, normally, with every impulse to a flexor to contract, goes an impulse to the corresponding extensor to relax. That is to say, such is the case if movement (in this case flexion) at the joint be desired. But if movement at a more distal joint be desired, or some other powerful resistance is to be overcome, flexors and extensors are simultaneously contracted, thus setting the joint firmly against movement, and enabling it to act as a necessary fixed fulcrum. The degree of rigidity depends upon the degree of simultaneous contraction. 
La característica más sobresaliente de la pedagogía moderna del piano es la importancia que se le ha dado a la relajación. Como resultado del amplio uso de este término, han surgido no pocas discrepancias y conceptos erróneos ${ }^{21}$ (Ortmann 1929: 59).

Una parte del cuerpo no está necesariamente en un estado de relajación o pasividad porque esté inmóvil; de hecho, a menos que descanse sobre un cuerpo exterior o se encuentre en otra forma de equilibrio estable, nunca está relajada²2 (Ortmann 1929: 89).

Schultz también da su punto de vista en el mismo sentido:

Yo creo que una interpretación incorrecta del significado de los movimientos altamente coordinados en general es la mejor explicación de la popularidad del principio de relajación. Un movimiento altamente coordinado es aquel que cumple ciertas exigencias mecánicas con un mínimo gasto de energía fisiológica. Esta economía de energía, sin embargo, es el resultado de una buena coordinación, no la causa. La escuela de relajación de la técnica pianística confundió el resultado con la causa, y ha intentado buscar una buena coordinación economizando energía. Errores de este tipo no son raros [... $]^{23}$ (Schultz 1949: 27-28).

Ortmann también interrelaciona el grado de respuesta sonora del instrumento con ataques de las teclas realizados con diferentes niveles de co-contracción muscular.

Estudios previos de la mecánica de la relajación y de la rigidez revelan que existe una estrecha correlación entre la relajación con un relativo bajo volumen sonoro, y entre una mayor rigidez con una relativa mayor intensidad del sonido²4 (Ortmann 1929: 241).

Después de leer las declaraciones de Ortmann y de Schultz, vemos, una vez más, que es fácil caer en la utilización de un lenguaje académico eventualmente poco adecuado para describir ciertos procesos que también transcurren en la ejecución guitarrística. Evitando demonizar la ausencia transitoria de relajación en algunos de los músculos implicados, cuya corrección depende más del momento justo en el que se aplique, que del hecho en sí mismo, y para facilitar una comprensión cabal, Charles Duncan ha preferido adoptar dos expresiones que consideramos ser más neutras y objetivas: tensión funcional y tensión disfuncional (Duncan 1980: 4-12). Desde que sabemos que es imposible tocar un instrumento musical sin algún tipo de tensión muscular, tal vez sea más práctico y adecuado, como forma de comunicar, utilizar la designación de Duncan para describir las acciones relacionadas con una ejecución eficiente y eficaz, gracias a su alta coordinación.

Por tanto, y volviendo al tema principal, podemos ver así que la estabilización de las articulaciones es producida por un cierto grado de tensión muscular, como antes hemos

21 Texto original: The salient feature of modern piano pedagogy is the stress placed upon relaxation. As a result of wide-spread use of this term not a few discrepancies and misconceptions have arisen.

22 Texto original: A part of the body it's not necessarily in a state of relaxation or passivity because it is motionless, in fact, unless it is resting upon some outside body, or is in another form of stable equilibrium, it is never relaxed.

23 Texto original: I believe, however, that a general misunderstanding of the significance of highly co-ordinated movements is the best explanation for the popularity of the relaxation principle. A highly co-ordinated movement is one which fulfils certain mechanical requirements with a minimal expenditure of physiological energy. This economy of energy, however, is the result of good co-ordination, not the cause. This economy of energy, however, is the result of good coordination, not the cause. The relaxation school of piano has mistaken the result for the cause. And has sought to produce good co-ordination by economizing energy. It is not an unusual error in kind.

24 Texto original: Previous study of the mechanics of relaxation and rigidity revealed the close correlation of relaxation with relative softness an of rigidity with relative loudness of tone. 
explicitado, pero también que el momento de su aplicación puede ser oportuno o no, dependiendo de las necesidades musicales y fisiológicas. Esta simple afirmación tiene grandes implicaciones en la optimización del esfuerzo realizado por el guitarrista. No obstante, asimilar la forma de optimizar los mecanismos y conseguir una respuesta espontánea puede representar grandes exigencias para el intérprete durante su formación, requiriendo una inversión de tiempo considerable. De ahí la importancia de que el instrumentista pueda tener una buena orientación técnica y teórica desde el principio de sus estudios.

\section{DIFUSIÓN Y ACEPTACIÓN DEL CONCEPTO}

Con relación a este asunto, en nuestro cuestionario preguntamos a los entrevistados si consideran que la teoría de las fijaciones/estabilizaciones articulares es ampliamente conocida y aceptada por la comunidad guitarrística contemporánea. Recibimos respuestas ilustrativas de la situación actual, que podemos ver en las siguientes citaciones.

Escande: Creo que es conocida en ciertos "bolsones" generados por los alumnos más consecuentes de Carlevaro, en algunas partes del mundo. Pero, en general, en lo que podría llamarse "la comunidad guitarrística" sigue habiendo una gran ignorancia acerca de las verdaderas ideas de Carlevaro. Mi propia práctica docente en muchas partes del mundo me ha demostrado cuánto interesa si se la explica a fondo y partiendo de su estructura lógica, y cuán tergiversada y "ninguneada" ha sido por parte de maestros que presumen de conocerla y nunca profundizaron en ella más allá de su envoltura exterior (Alfredo Escande, comunicaciones personales por e-mail, 10 de junio y 24 de agosto, 2018).

Fernández: En el ámbito "local" (Río de la Plata) me parece que sí. Está lejísimo de ser ampliamente conocida en la comunidad guitarrística internacional, y mucho menos aceptada. Siguen prevaleciendo los conceptos de Tárrega-Pujol (apoyado-no apoyado como una dicotomía) y en lo personal, cuando he intentado explicar el tema, hay dificultades grandes para su comprensión, porque para entender el papel de la fijación es necesario repensar toda la relación del guitarrista con su cuerpo, y toda la acción física necesaria para la ejecución, desde un punto de vista consciente. Es difícil revertir lo aprendido, y en el marco de una clase magistral, con tiempo limitado, prácticamente imposible (Eduardo Fernández, comunicación personal por e-mail, 8 de agosto, 2018).

Basándonos en los comentarios anteriores y además en nuestra propia experiencia profesional, podemos confirmar que no solamente existe bastante ignorancia al respecto, como también algún desdén y menosprecio, tal vez por falta de conocimiento serio de la mecánica de la estabilización articulary su utilización en la interpretación instrumental. Sin menoscabo de lo anterior, también debemos considerar que los instrumentistas que llegan a una clase magistral o leen un libro de formación avanzada, normalmente ya tienen varios años de estudios a cuestas y lo que buscan con esto es eliminar las lagunas que han tenido en su aprendizaje, sea cual sea su origen. Por tanto, estos guitarristas no tienen por qué ser prosélitos de una escuela para hacer suyo cierto mecanismo aislado que han asumido como útil para mejorar su técnica y promover su evolución personal, ya que la formación de un guitarrista es acumulativa, y cuentan todas las experiencias vividas. Recordamos que en una clase magistral de Carlevaro a la que asistimos ${ }^{25}$, él dijo: "Ojalá yo hubiera tenido un Carlevaro como profesor". No obstante, si Carlevaro no hubiera tenido los estudios técnicos y musicales que tuvo, previos a su autoformación, tal vez no habría llegado a ser el gran

25 Realizada en la sede de la SGAE, Madrid, 1990. 
guitarrista y pedagogo que fue, ni hubiese dejado el valioso conjunto de herramientas para propiciar la superación de los guitarristas que hoy conocemos. Aunque algunas personas hayan aceptado las enseñanzas carlevarianas como un dogma, no lo son, antes, lo contrario.

Ahora, otra cuestión es: ¿por qué esta forma de ver la técnica guitarrística, aunque ha tenido una expansión geográfica considerable, no ha prosperado más en el ámbito mundial?26 Según el testimonio de Gimeno, es probable que el propio Carlevaro no haya adoptado las actitudes adecuadas ni tomado las acciones más eficaces para que ello sucediera.

¿Quién es responsable? Siento que, si su escuela no ha tenido el verdadero reconocimiento, es por el mismo maestro Carlevaro (Magdalena Gimeno, comunicaciones personales por e-mail, 13 y 14 de septiembre, 2018).

Sin embargo, Solari, tiene un punto de vista muy diferente, y achaca su relativamente limitada difusión y aceptación a cuestiones políticas y sociales:

[...] la comunidad guitarrística en su gran mayoría sigue anclada en un concepto guitarrístico y musical vetusto. Carlevaro además nació en el sur del mundo y allá se quedó. El racismo, el imperialismo y la colonización cultural eurocentrista y "occidental" no perdonan y la guitarra no constituye una excepción (Solari 2018).

Es probable que tanto Gimeno como Solari tengan razones válidas para hacer estos comentarios. Sin embargo, vemos que el libro de Charles Duncan (1980), publicado en inglés en Estados Unidos, cuyo autor es un ciudadano del primer mundo que, entre otros asuntos, también abordó la problemática de la fijación de forma pormenorizada en el mismo momento histórico que Carlevaro, pero utilizando otros argumentos, tampoco ha tenido la repercusión que se pudiera prever en la comunidad guitarrística internacional, a pesar de la buena calidad de su trabajo. En comunicaciones personales por e-mail con este autor (15 de abril de 2015), en donde le manifesté el propósito de realizar una traducción al portugués de su obra, Duncan nos dejó ver que no era fácil ver su libro traducido a otros idiomas, aunque ya había habido algunas tentativas. Entonces, teniendo en consideración estos hechos, tal vez haya más motivos que los esgrimidos por los guitarristas mencionados.

Esta desidia también podría radicar en la manera de enfocar la ejecución instrumental como una mera destreza, en la que el trabajo intelectual tendría una expresión mínima, y, por tanto, cuestiones conectadas con disciplinas como la anatomofisiología, la ergonomía, la física, o las matemáticas no serían bien aceptadas, por no ser aparentemente redituables para una función asumida solo como práctica, especialmente por la faceta de instrumento popular que parcialmente tiene la guitarra. Pero sobre todo, lo que podemos constatar es que, a pesar de toda la herencia de la Ilustración, todavía persiste una no despreciable resistencia al estudio razonado y a la utilización consciente y voluntaria de determinados mecanismos no tradicionales, aunque esto sea probadamente eficaz. Por supuesto que este no es un fenómeno nuevo, como se puede extraer de las siguientes observaciones de Fernando Sor, en su Método para guitarra de 1830:

Alguien me dirá: 'no valía la pena decirme lo que ya hago sin pensar, ni utilizar, para explicarlo, este razonamiento matemático'. Se puede hablar una lengua, y a veces bastante bien, sin necesidad de ser gramático, pero cuando el objetivo es examinar los principios de su organización, es imprescindible recurrir a la gramática, y sobre todo a la lógica si la finalidad es enseñarla, ya que

26 En un trabajo posterior, sería importante dar continuidad y ampliar el rango de aplicación de las entrevistas, para comprobar que, en efecto, no se conoce este concepto en otras latitudes. 
sin una buena clasificación de las ideas no puede haber claridad ni precisión en la explicación (Sor 2008: 58).

Por otra parte, tal vez sirva de consuelo para los defensores del mecanismo que nos ocupa saber que alrededor de 1940 ya existía resistencia ante el uso del término fijación en el piano, presente en la posición pedagógica del pianista inglés Tobias Matthay, de quien hemos hablado anteriormente, autor del libro The visible and invisible in piano technique. A digest (1932 y 1947), entre otros escritos. Los exacerbados comentarios de Matthay en contra de la utilización del término fijación nos hacen ver que ya por aquella altura estaba instalada ampliamente la polémica entre los profesores de piano de su tiempo. Schultz, en un capítulo entero de su libro dedicado a Matthay, y hablando del mismo, dice que "de hecho, se podría juzgar que la mera idea de tal fijación lo llenaría de una especie de horror" (Schultz 1936: 242), como se puede corroborar en las siguientes citaciones, que pueden ser entendidas como una crítica a Ortmann y a Schultz, quienes eran favorables al uso de las fijaciones:

[...] algunos de los pedagogos más antiguos; desafortunadamente, fueron bastante vagos y poco claros en cuanto a lo que significaba fisiológicamente; de ahí surgió su idea errónea de la "FIJACIÓN" - ¡fijación de las articulaciones durante el momento del ataque! Un terrible error, que provoca una rigidez paralizante de todo el miembro, lamentablemente copiado por algunos autores recientes ${ }^{27}$ (Matthay 1947: 20).

Matthay, así como Wheatley-Brown, a quien citamos anteriormente, también se refería críticamente al lenguaje inexacto e inconsistente utilizado por algunos docentes a mediados del siglo pasado.

[...] un término mal elegido puede causar mucho daño. Como se indicó anteriormente, "Fijación" es un término funesto por naturaleza, ;ya que es probable que sugiera todo lo que no se debe hacer al tocar! [...] Mientras que, como se demostró, lo que se necesita, es, una estabilización momentánea totalmente efectiva pero elástica de esas bisagras (o articulaciones), cuando sea necesario durante el momento de dar impulso a la tecla 28 (Matthay 1947: 119).

Matthay, en sus comentarios, también se refiere a otra denominación: "anclaje" (anchorage), al parecer habitual en el lenguaje técnico de algunos pianistas coetáneos, que también identificaría, en el mejor de los casos, a la estabilización articular.

Hablar de Anclajes o Fijaciones a este respecto, es solo una forma vaga y engañosa de denominar al simple hecho de que ciertas partes de los miembros incluidas en la ejecución instrumental deben ser (invisiblemente) tensadas o relajadas, apenas como una forma de ofrecer una Base o Apoyo estable y seguro para permitir el esfuerzo y el movimiento en otras partes. Aquí se necesitan afirmaciones definidas sobre qué partes de un segmento de un miembro se deben dejar pasivas o estabilizadas, y cuáles deben realmente actuar. Solo eso nos ayudará. [...] El término "Fijación" debería, por lo tanto, desterrarse estrictamente del vocabulario del Pianista. Es incalculablemente

27 Texto original: [...] some of the older pedagogues; unfortunately, they were quite vague and unclear as to what it meant physiologically; hence arose their mistaken idea of "FIXATION" - fixation of the joints during the moment of touch! A terrible mistake, leading to a paralyzing stiffening of the whole limb, unhappily copied by some recent authors.

28 Texto original: [...] an ill-chosen term may do much harm. As indicated earlier, "Fixation" is a baneful term of this nature, since it is likely to suggest everything one should not do in playing! [...] Whereas, as demonstrated, what is needed, is, momentarily, a fully effective but elastic steadying of those hinges (or joints), when needed during the moment of key-propulsion. 
dañino; y el "anclaje", aunque no tan malignamente sugerente, es un poco mejor; mientras que, como se señaló, ambos son bastante desinformativos en cuanto a la causa del bien hacer [...] $]^{29}$ (Matthay 1947: 24).

Los últimos fragmentos citados son bastante elocuentes. Sin embargo, detrás de un velo de rechazo absoluto, en las opiniones de Matthay no parece haber tanta discordancia con un recurso técnico útil, que a veces es identificado erróneamente como fijación, como con su "maligna" denominación y con su uso "dañino" e inadecuado, porque, en realidad, este debería haber sido llamado estabilización.

\section{CONSIDERACIONES FINALES}

Poco menos de un siglo después de la publicación de los escritos de los pianistas que hemos citado, podemos leer algunos comentarios, tal vez "resignados", del musicólogo Luca Chiantore en La historia de la técnica pianística (2016), en torno al destino de la bibliografía acerca de la técnica pianística con argumentación científica, que tuvo gran auge durante la primera mitad del siglo XX. Después de más de setecientas páginas, en las que este musicólogo analiza profundamente la obra de numerosos músicos de tecla y compositores en el transcurso de cinco siglos, el estudioso cierra su trabajo diciendo:

Pero ¿qué queda de tanta «ciencia» en la enseñanza de los pedagogos de hoy? Algunas ideas generales, sin duda, y un vocabulario básico, al que acuden diariamente pedagogos de todo el planeta [...] La clase de piano sigue estando marcada por la corrección del detalle y por un análisis de los problemas concretos de cada alumno, en el cual la experiencia personal del pedagogo prima sobre el conocimiento teórico de la técnica (Chiantore, 2001: 722).

Sin desmerecer las observaciones de Chiantore, nos gustaría matizar un poco su significado. Pensamos que sus frases podrían ser entendidas equívocamente como desdeñosas en relación con el estudio teórico de un instrumento desde una perspectiva científica de la técnica. En realidad, Chiantore, quien habla desde el conocimiento, está dejando de manifiesto que, gracias a todos esos estudios publicados acerca de la mecánica de la ejecución apoyados en la ciencia, la técnica del instrumento ha llegado a altísimos niveles. Como el conocimiento teórico de la técnica ha sido una vía para facilitar la organización mental del trabajo físico y para optimizar la interpretación artística, el músico actual se ha visto libre de resolver muchos problemas, porque gran parte de ellos fueron solucionados gracias a un enfoque científico de la técnica instrumental, especialmente en los últimos dos siglos. De hecho, el estudio del camino recorrido para llegar a este punto parece estar hoy primordialmente reservado al musicólogo, al investigador, o simplemente al músico curioso.

Actualmente es relativamente fácil acceder al conocimiento de los mecanismos útiles, tal como a las formas de adquirirlos y asimilarlos, aunque no haya unanimidad en su forma de aplicación. Consecuentemente, muchos instrumentistas no necesitan conocer profundamente aquellos estudios escrupulosos que les precedieron para poder utilizar

29 Texto original: To talk of "Anchorages" or "Fixations" in this connection, is only a vague and misleading way of stating the plain fact, that a certain portion of our playing-limb must either be (invisibly) exerted or left lax, so as to form a stable and secure Foundation or Basis for the exertion or movement of other portions. Definite statements are here needed: as to which portions of a limb to leave passive or supported, and which to exert. That alone will help us. [...] The term "Fixation" should therefore be strictly banished from the Pianist's vocabulary. It is incalculably harmful; and "anchorage," though not so evilly suggestive, is little better; while, as pointed out, both are quite uninformative as to the cause of well doing [...]. 
los recursos más adecuados y eficaces para dominar la interpretación, pues ellos se han formado integrados en una cultura de bien hacer que ha acumulado conocimiento especulativo y práctico pertinente a lo largo del tiempo. Por tanto, no existe la necesidad de que los estudiantes orientados por un docente bien preparado tengan que conocer todos los estudios científicos precedentes para obtener buenos resultados musicales, ya que su mentor podrá guiarlos por los caminos más adecuados.

Asimismo, Chiantore afirma que "cualquier movimiento, una vez asimilado a nivel muscular, puede realizarse sin indecisiones, olvidando que cuando tocamos no hemos de pensar en el gesto, sino en la sonoridad resultante" (Chiantore 2001: 723).

Concordamos con Chiantore hasta cierto punto, teniendo presente la importancia de la previa automatización de una amplísima serie de acciones útiles, a las que Carlevaro llama "hábitos positivos", para que eso suceda espontáneamente. Entre los hábitos positivos estaría el gobierno de las estabilizaciones gracias a una perfecta coordinación muscular y al control de la tensión funcional durante la ejecución. Pero, sin duda, uno de los objetivos primordiales de un músico es conseguir la interpretación artística deseada a partir de una idea o de una imagen mental y que la música fluya naturalmente durante la ejecución, como sostiene el pianista György Sandor en su interesante libro On Piano Playing: Motion, Sound and Expression (Sandor 1981).

Para finalizar, consideramos importante recalcar que, aunque las dificultades técnicas puedan ser superadas mediante el desarrollo de las habilidades físicas individuales, los mecanismos aprendidos basándose exclusivamente en el pragmatismo y en la intuición serán de difícil sistematización y transmisión en el caso de no poseer una teoría que sirva para organizarlos. A pesar de que ciertos problemas técnicos de los músicos instrumentistas puedan ser resueltos por medio del instinto, de la intuición o del conocimiento empírico adquirido mediante los aciertos y de los fallos, la falta de una observación metódica que permita desarrollar una teoría que revele patrones orientadores acarreará, a menudo, retrasos, situaciones incomprensibles, y hasta problemas físicos derivados de esfuerzos excesivos innecesarios. Consiguientemente, será importante tener en cuenta que la capacidad de diagnosticar el origen de las dificultades gracias al conocimiento profundo de los mecanismos puede ser fundamental en las habilidades de quienes enseñan a tocar un instrumento, aunque a veces haya cierta resistencia ante los cambios de paradigma que representan las propuestas pedagógicas más recientes.

\title{
REFERENCIAS BIBLIOGRÁFICAS
}

\author{
Aguado, Dionisio \\ 1843 Nuevo Método para Guitarra. Reimpresión facsimilar (1994). Madrid. Heidelberg: Chanterelle \\ Verlag. \\ BARCELó, RicARDo \\ 2000 Adiestramiento técnico para guitarristas. Madrid: Real Musical. \\ 2015 O Sistema Posicional na Guitarra. Saarbrücken: Novas Ediciones Académicas. \\ Carlevaro, Abel \\ 1969-1975 Serie didáctica. Cuadernos 3 y 4. Buenos Aires: Barry. \\ 1979 Escuela de la Guitarra. Buenos Aires: Barry. \\ Chiantore, Luca \\ 2016 Historia de la técnica pianistica. Un estudio sobre los grandes compositores y el arte de la interpretación \\ en busca de la Ur-Technik. Madrid: Alianza Editorial (1 ${ }^{\mathrm{a}}$ ed. 2001).
}


Duncan, Charles

1980 The art of classical guitar playing. Miami: Summy-Birchard Music.

Escande, Alfredo

1996 "La Escuela de Carlevaro" [conferencia]. Cabildo de Montevideo (6 de septiembre) disponible en: www.elguitarrista.net/articulos/la_escuela_de_carlevaro.html [acceso: 11 de agosto de 2019].

2005 Abel Carlevaro. Un nuevo mundo en la guitarra. Montevideo: Aguilar/Santillana.

2019 Cincuenta años de entrevistas a ABEL CARLEVARO 1951-200. Disponible en: file:/ / / C:/Users/ rican/Downloads/Carlevaro-Entrevistas\%201951-2001.pdf [acceso: 11 de agosto de 2019].

FERNÁNDEZ, EduARdo

2000 Técnica, Mecanismo, Aprendizaje: una investigación sobre llegar a ser Guitarrista. Montevideo: Ediciones ART.

2016 "18 instantáneas de Abel Carlevaro" [6] (3 de enero). Disponible en: https://www.edufernguitar.com/single-post/2016/01/03/18-instant\%C3\%A1neas-de-Abel-Carlevaro [acceso: 11 de agosto de 2019].

Gowitzke, Barbara y Morris Milner

1999 El cuerpo y sus movimientos. Bases científicas. Barcelona: Editorial Paidotribo.

Jiménez Muñoz, Antonio José

2013 "Abel Carlevaro: florecimiento de una nueva técnica instrumental en el s. XX", Sexto Orden 8, pp. 19-33. Disponible en https://issuu.com/sexto.orden/docs/no8 [acceso: 11 de agosto de 2019].

MatThay, Tobias

1947 The visible and invisible in piano technique A digest. $2^{\mathrm{a}}$ edición revisada (1 ${ }^{\mathrm{a}}$ ed., 1932). Nueva York: London, Humphrey Milford Oxford University Press.

Ortmann, Отto

1929 The phisiologycal mechanics of piano technique. Hertford: Stephen Austin \& Sons, Ltd.

Primus, Oliver y PATrick Zeoli

1997 [video] A Guitar Lesson with Abel Carlevaro. Winterthur, Suiza. [Hugorowsky] 9/2/2013 YouTube. Disponible en: www.youtube.com/watch?v=-XbUkcjuMds [acceso: 11 de agosto de 2019].

SANDOR, GYÖRGY

1981 On Piano Playing: Motion, Sound and Expression. Nueva York: Schirmer Books Macmillan Publishing.

SCHultz, ARnold M.

1949 The Riddle of the Pianist's Finger and Its Relation to a Touch Scheme. Nueva York: Schirmer $\left(1^{\mathrm{a}}\right.$ ed. 1936).

SOR, FERNANDO

2008 Método para guitarra. E. Baranzano y R. Barceló (traductores). Fafe: Editora Labirinto (1 ${ }^{\mathrm{a}}$ ed. París, 1830).

Wheatley-Brown, Michèle

2011 "An Analysis of Terminology Describing the Physical Aspect of Piano Technique". Tesis de máster. Ottawa: Universidad de Ottawa. 